\title{
Linked Data en Repositorios Digitales de Latinoamérica.
}

\author{
Nelson Piedra ${ }^{1}$, Janneth Chicaiza ${ }^{1}$, Elizabeth Cadme, Jorge López ${ }^{1}$, Diana Torres \\ ${ }^{1}$ Edmundo Tovar ${ }^{2}$ \\ nopiedra@utpl.edu.ec,jachicaiza@utpl.edu.ec, iecadme@utpl.edu.ec, \\ jalopez2@utpl.edu.ec, datorres@utpl.edu.ec, edmundo.tovar@upm.es \\ ${ }^{1}$ Universidad Técnica Particular de Loja, San Cayetano Alto S/N, 11016o8, Loja, Ecuador. \\ 2 Universidad Politécnica de Madrid, Avda. Montepríncipe S/N, 2866o, Madrid, España.
}

\begin{abstract}
Resumen: La integración de información, la ejecución de tareas de analítica de big data en entornos federados, así como el re-uso y adaptación de recursos educativos abiertos plantea desafíos e términos de mejorar la interoperabilidad e integración de información. El enfoque de linked data potencia la interoperabilidad e integración en un contexto distribuido y de alta heterogeneidad como es la Web. El propósito de este trabajo es proporcionar información relevante de repositorios digitales usando estándares abiertos, centrados en el enfoque de la Web Semántica y basados en tecnologías de Datos Enlazados. La aplicación de esta propuesta resulta en un ecosistema semántico que contribuye con la interoperabilidad, integración, navegación, la búsqueda y enlazado semántico de repositorios digitales y de recursos educativos abiertos en el contexto de Latinoamérica.
\end{abstract}

Palabras-clave: Repositorios Digitales, Datos Enlazados, Web Semántica, RDF, OAI-MPH, Ontología, Linked Data, Open Data

\section{Introducción}

En los primeros días de la Web, las cosas eran fascinantes y nuevas, los usuarios estaban emocionados con la posibilidad de navegar a través de sitios que ofrecían grandes colecciones de documentos hiper-enlazados, distribuidos en el mundo. Inicialmente esto fluía unidireccionalmente, las usuarios accedían a la información y la usaban en una variedad de vías.

Hoy, la Web se ha vuelto mucho más interactiva. Hay nuevas expectativas acerca de como consumir la información disponible, en actividades de búsqueda en la Web, integración de información y actividades de minería de datos en la Web. Esto se deriva en primer lugar por el creciente número de empresas alineadas con la tendencia de aumentar las posibilidades de creación de contenidos generados por los usuarios. Específicamente, en lugar de solo leer contenidos, las personas están 
usando la Web para crear contenidos y para interactuar con otros a través de plataformas Web de redes sociales. Incluso si las personas no tienen interés en crear contenidos o en interactuar, la evolución de la Web sigue cambiando las actividades humanas.

En segundo lugar, las capacidades en desarrollo Web han cambiado enormemente. Ya no solo se crean sitios Web con contenidos estáticos, sino sitios en los que se ejecutan transacciones complejas. En tercer lugar, cada vez son más las organizaciones que publican en sus sitios Web contenidos en formatos estructurados con el objetivo de compartirlos con quienes se interesen en reusar estos datos y desarrollar transacciones en línea, o nuevos servicios.

Estas tendencias dejan ver algunas cuestiones críticas en la Web. La mayoría del contenido Web está diseñado en formatos entendible por los seres humanos, y no para que los programas de computación puedan manipular de manera fiable la semántica de este contenido. El actual enfoque de Web Semántica busca incorporar estructuras semánticas (esto es dotar a la información de significados bien definidos) a la información de las páginas Web, de manera que se construya un entorno en el que los agentes software pueden ejecutar colaborativamente tareas de procesamiento sobre los datos que actualmente se limitan a mostrar.

Alcanzar el propósito de la Web Semántica depende de la transformación de la Web actual como un espacio de documentos hiper-enlazados que se muestran a un lector humano, en un red global de datos estructurados asociados a descripciones de cosas reales o abstractas, que en el caso de este trabajo, pueden incluir a registros bibliográficos (trabajos, manifestaciones y expresiones), autores, organizaciones, tópicos, información geográfica, entre otros.

Este trabajo plantea una vía para mejorar la integración de información sobre repositorios digitales abiertos usando un enfoque de Web Semántica. Se busca añadir a los repositorios digitales actuales, significados comprensibles (o "semántica") en formatos procesables automáticamente por agentes máquina. Se describe el caso de aplicación del marco de trabajo propuesto en (Piedra et al., 2015), para la publicación de los datos extraídos de repositorios digitales latinoamericanos que usan OAI-PMH; el objetivo es apoyar la creación de un ecosistema Web en la que sea factible y eficiente la integración automática datos.

\section{Trabajos Relacionados}

Los Recursos Educativos Abiertos (en inglés: Open Educational Resources, OER), son materiales educativos digitales libres que pueden ser usados, compartidos y editados por profesores, estudiantes y autodidactas. Hay cientos de proveedores de OERs que comparten de forma abierta recursos educativos a través de Internet. La interoperabilidad entre estas fuentes heterogéneas y diversas de OERs es una 
herramienta poderosa para alcanzar los objetivos planteados por el movimiento de OER: ampliar el acceso, reuso y remezcla de materiales educativos.

Internet genera un escenario global en el que las condiciones de interoperabilidad (IEEE, 1990) son las que garantizan el descubrimiento, la distribución y re-uso de recursos digitales, más allá de condiciones de gestión local, de modelos tecnológicos y de herramientas de cualquier tipo. Para lograr la interoperabilidad entre repositorios de librerías digitales la Iniciativa de Archivos Abiertos (OAI) propone el protocolo para la recolección de metadatos denominado OAI-PMH (OAI, 2015). OAI-PMH se basa en estándares abiertos, por tanto, garantiza la interoperabilidad automática entre emisores y receptores de recursos digitales, con independencia del software utilizado e incentivando la neutralidad tecnológica y la innovación (ver Figura 1).

Un escenario de integración hace posible la combinación de recursos de información existentes en diversas fuentes, esto proporciona al usuario una vista unificada de dichos recursos y también puede actuar como una fuente de datos para diversas aplicaciones (Lenzerini, 2002). Para conseguir este propósito, primero se deben resolver problemas de heterogeneidad a nivel de: (a) repositorios, (b) formatos de datos, (c) esquemas de metadatos, y (d) vocabularios o diccionarios de datos. Para abordar cada una de estas dimensiones, existen diferentes propuestas. El grado más bajo de interoperabilidad (a nivel de repositorios) se consigue con protocolos como OAI-PMH, el cual facilita la extracción e intercambio de metadatos, aunque su uso no asegura el reuso e integración de la información. Para asegurar la interoperabilidad semántica entre diferentes colecciones o esquemas (Gendt et al. 2006 y Francesconi et al. 2008) proponen crear links entre objetos equivalentes. En este sentido, el uso de vocabularios abiertos y la generación de datos abiertos en formatos legibles para máquinas, pueden ser claves para integrar elementos de diferentes repositorios, más allá de estándares y acuerdos previos entre los proveedores. En (Piedra et al., 2014), se plantea una aproximación basada en Linked Data para mejorar la integración de repositorios de OpenCourseWare, un tipo de OER. En (Zengenene et al. 2014) se propone un marco teórico enfocado a proyectos de librerías digitales para publicar sus metadatos mediante un enfoque de datos enlazados. Otros casos recientes de generación de datos enlazados son presentados en trabajos como (Malakhov et al., 2014, Anibaldi et al. 2015 y Sztyler et al., 2014).

Aunque OAI-PMH facilita el intercambio de metadatos en la Web, aún pueden persistir problemas para integrar los datos extraídos desde diversos repositorios. En un entorno abierto como la Web, no es posible estandarizar los procesos de descripción y publicación de metadatos, por tanto, cada institución puede manejar diferentes formatos de datos o esquemas de metadatos o vocabularios. Se requiere un enfoque de Web Semántica. En (Piedra et al., 2015), los autores proponen un Marco de trabajo para la Integración de Recursos Digitales Basado en un enfoque 
de Web semántica, siguiendo los principios de Linked Data y el ciclo de vida para la publicación de datos enlazados.

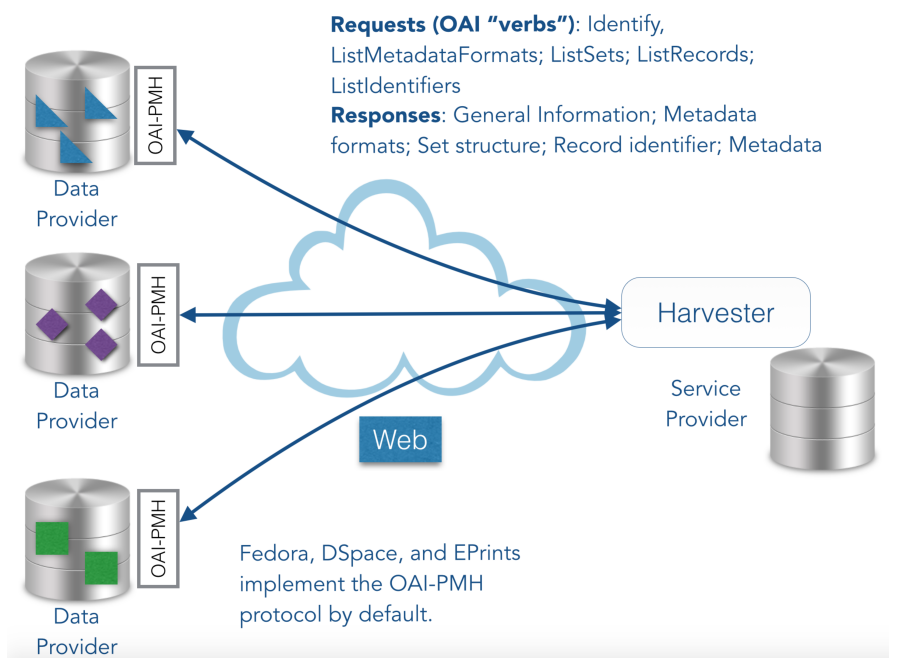

Figura 1-Aproximación de interoperabilidad usando OAI-PMH. La Web es la infraestructura de transporte de datos y metadatos

\section{Integración de recursos digitales en la Web Semántica}

La Web Semántica visionada por Berners-Lee (2001) añade a la Web de Documentos, el significado que le hace falta para disponer de un entorno en el que sea posible acceder a los datos contenidos en sitios Web. Esta visión implica que los datos están almacenados en una base de datos global distribuida (Heath \& Bizer, 2011). Por lo tanto será posible procesar automáticamente la información de un modo más exacto y completo.

La publicación de Datos Enlazados se fundamenta en cuatro principios básicos de diseño propuestos por Tim Berners-Lee (2006): (1) Usar URIs para identificar los recursos de la Web. (2) Usar URIs-HTTP para que los usuarios puedan localizar y consultar estos recursos. (3) Proporcionar información útil acerca del recurso cuando la URI haya sido consultada, utilizando $\mathrm{RDF}^{1}$ para describir recursos y SPARQL $^{2}$ para consultarlos. (4) Incluir enlaces a otras URIs relacionadas con los datos contenidos en el recurso, de forma que se potencie el descubrimiento de información en la Web.

1 Resource Framework Description, es un lenguaje para representar y publicar datos estructurados en la Web.

${ }^{2}$ SPARQL es el lenguaje para consultar grafos modelados con RDF. 
En un contexto basado solamente en OAI-PMH, la Web es la infraestructura de transporte de datos y metadatos (Ver Figura 1), mientras que desde Linked Data, los datos y su semántica son parte de la misma Web. Los recursos digitales están identificados a través de URIs y descritos en un formato que permite la lectura y procesamiento automático por parte de agentes máquina (Ver Figura 2).

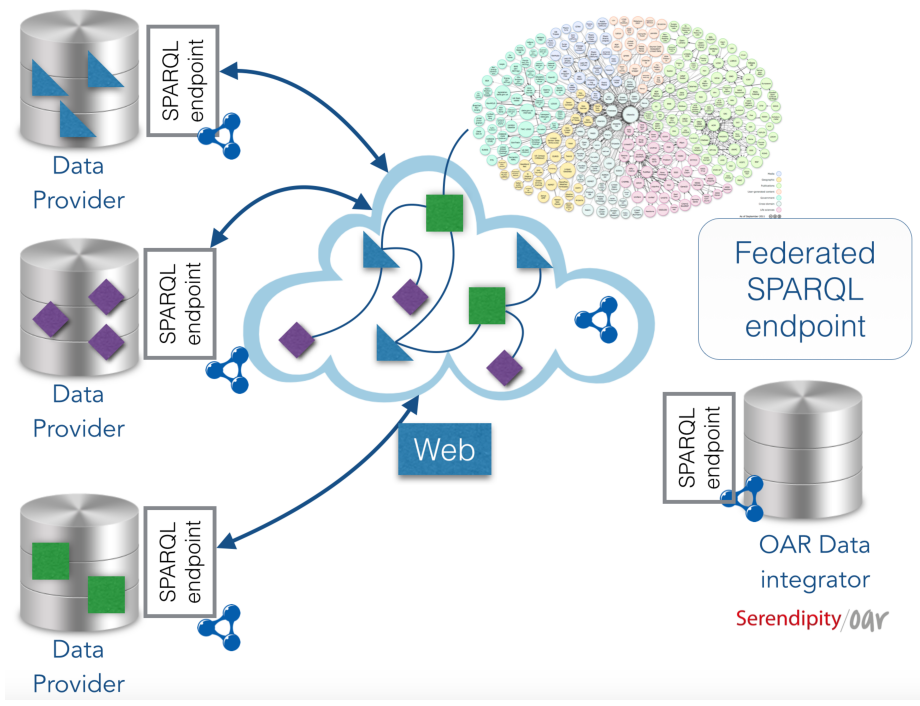

Figura 2 - Integración e interoperabilidad de metadatos bibliográficos de recursos digitales a través de Linked Data. Los datos y los metadatos son parte de la misma Web.

\section{Marco de Trabajo para la Publicación de Datos Enlazados.}

El ciclo de publicación de datos enlazados comprende una serie de componentes y actividades interrelacionadas. En (Piedra et al., 2015), se presenta el marco de trabajo usado para de extracción de metadatos vía OAI-PMH y su publicación como datos enlazados.

1. Selección de fuentes de datos, se han seleccionado repositorios que tienen habilitado el protocolo OAI-PMH. Esto crea una Federación de Librerías Digitales. A continuación se listan las 154 proveedores de que son parte del alcance de este trabajo:

ACBSC, ADB, BANREPCULTURAL, CAB-CNEA, CEDIA, CEPAL, CES, CIDE, CLACAI, CLACSO, COLCIENCIAS, CONICYT, CTI-BR, EAFIT, EAN, EDUCACION-AR, EIA, EMBRAPA, EMBRAPA, ENAP, ESPE, ESPOCH, ESPOL, FGV, FIOCRUZ, FLACSOANDES-EC, FUMEC-BR, FUNDE, FURG, IAEN, IBICT, IBICT, ICESI, IMARPE, INPE, INT, IPN, ITESO, JAVERIANA, LAMOLINA, LASALLE, LASALLISTA, MARINGA, MDP, MEC-BR, METABIBLIOTECA，POLIGRAN，PUC-RIO，PUCE，PUCESI，PUCP, 
CBIE-LACLO 2015

Anais dos Workshops do IV Congresso Brasileiro de Informática na Educação (CBIE 2015)

PUCRS, REPOSITORIUM, SENADO-BR, SENECYT, SLD, UAEH, UAEMEX, UANL, UAO, UASB, UAZUAY, UBA, UBA, UC, UCA, UCAB, UCARABOBO, UCCOR, UCE, UCES, UCHILE, UCR, UCSG, UCUENCA, UDLA, UDO, UEB, UEPG, UERJ, UFASTA, UFES, UFGD, UFLA, UFMA, UFMS, UFOP, UFPA, UFPE, UFPR, UFRB, UFRGS, UFS, UFSC, UFSC, UFU, UFVJM, UG, UIDE, ULA, UNA, UNAB, UNAB, UNAD, UNAL, UNAL, UNAM, UNAM, UNAM, UNAM-AR, UNB, UNC, UNCU, UNED-CR, UNEMI, UNESP, UNESP, UNIANDES-CO, UNICAMP, UNICEUB, UNIMILITAR, UNINORTE, UNISABANA, UNJBG, UNL, UNL, UNLP, UNLP, UNLP, UNMSM, UNR, UNSA, UPC, UPEC, UPN, UPR, UROSARIO, USAT, USB, USCS-BR, USFQ, USP, USP, UTA, UTALCA, UTC, UTFPR, UTMACHALA, UTN, UTP, UTPL, UV-MX, UWI, WORLDBANK,

2. Cosecha de metadatos desde repositorios: El protocolo OAI-PMH es la base del modelo de cosecha de metadatos de recursos digitales.

- Uso de librería Harvester 2.0 para extraer los metadatos de los repositorios, a través del protocolo OAI-PMH, usando el verbo "listRecords".

- Los metadatos extraídos se almacenan en una base de datos relacional en forma de tripletas.

- Limpieza de los datos extraídos. El objetivo es detectar y corregir datos corruptos o erróneos. El proceso consiste en analizar patrones inconsistentes en los datos y ejecutar un esquema de limpieza. Se eliminan problemas de ambigüedad en los tópicos añadidos a cada recurso digital.

3. Modelado del vocabulario. En el núcleo de la Web Semántica están las ontologías. Las ontologías son la vía para describir el mundo que nos rodea. Las ontologías y los vocabularios abiertos constituyen el esquema base a partir del cual se describen los recursos y entidades de la Web. Los datos adquieren significado a través de ontologías, que se han ido construyendo gradualmente de acuerdo las necesidades de cada dominio de conocimiento. Por lo tanto, en cierto sentido y con las limitaciones del desarrollo tecnológico actual, al estructurar los datos contenidos en silos de información Web, estos pueden ser "entendidos" y procesados automáticamente en tareas que busquen hacer tareas complejas de re-uso e integración de información almacenada en sitios web distribuidos y heterogéneos. El re-uso de recursos ontológicos y no-ontológicos es clave para incrementar el grado de interoperabilidad en el escenario de Linked Open Data propuesto.

4. Conversión de datos a formato RDF:

- Identificación de recursos a través de URIs persistentes. Se han diseñado dos tipos de URIs: 1) para identificar los componentes del vocabulario (clases, propiedades y relaciones); y, 2) para describir el material bibliográfico.

- Conversión de datos extraídos a formatos estándar, abiertos e interoperables de la Web Semántica, de manera que se contribuya a facilitar su acceso y reutilización, y se resuelva el problema de disponer de recursos digitales aislados en silos (Ver Figura 3). 
CBIE-LACLO 2015

Anais dos Workshops do IV Congresso Brasileiro de Informática na Educação (CBIE 2015)

- Limpieza de datos generados. Las actividades buscan reducir la ambigüedad y purgar la información extraída y generada durante el proceso de conversión.

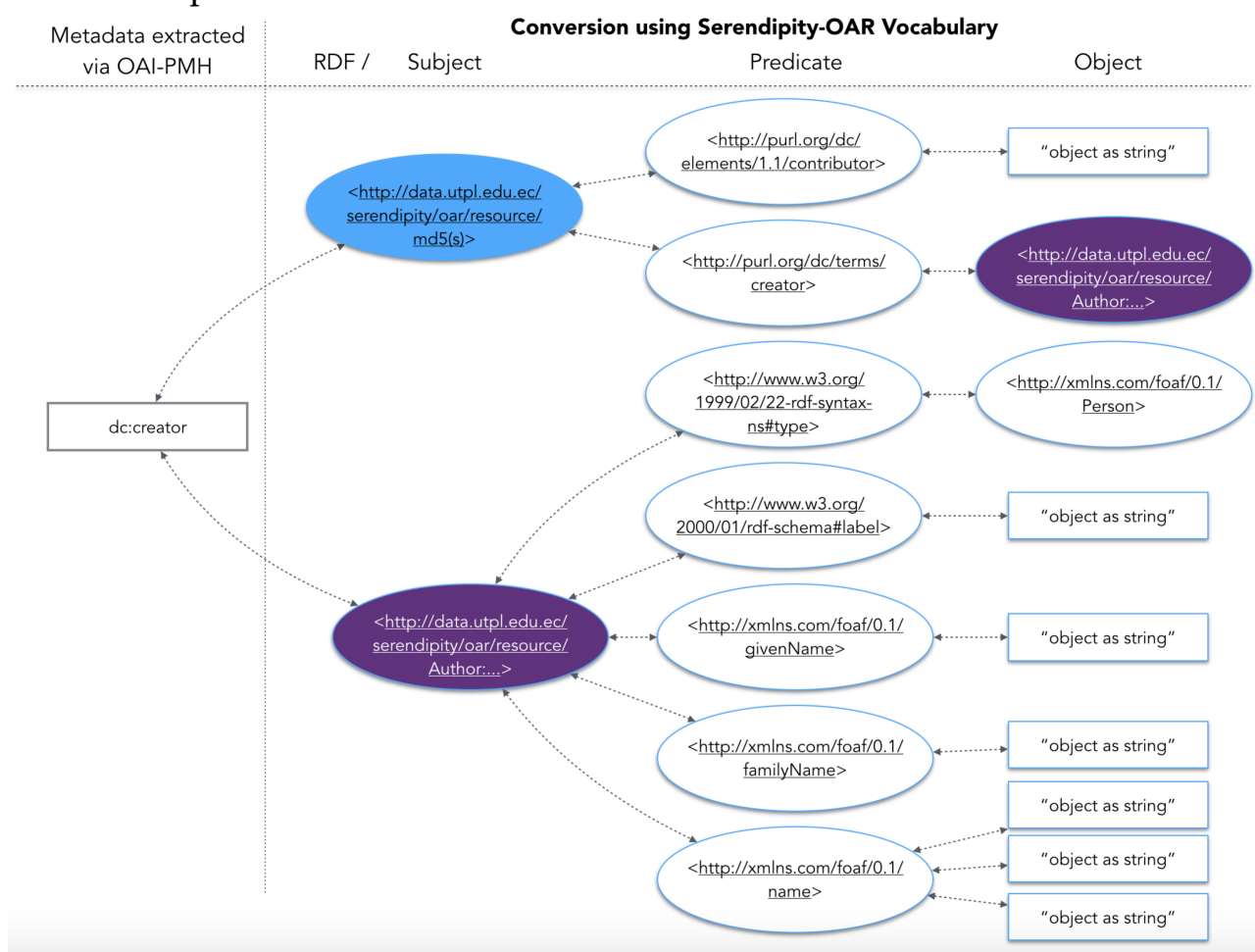

Figura 3 - Mapeo de creadores de recursos digitales, de metadata extraída desde repositorios OAI a tripletas basadas en Ontología propuesta.

5. Enlazado de datos a través de sus relaciones semánticas. Para mejorar el descubrimiento de los recursos es necesario crear enlaces RDF hacia recursos externos publicados en la nube de Linked Open Data y con datos de repositorios externos. Se han enlazado los tópicos y palabras claves de cada recurso digital con el contenido de vocabularios controlados y esquemas de clasificación. En particular con Nomenclatura de la UNESCO, y con DBpedia.org y DBpedia Latinoamérica (http://es-la.dbpedia.org/sparql).

6. Publicación y Explotación de datos. La publicación de datos abiertos enlazados en la Web mediante tecnologías estándar propuestas por la $\mathrm{W}_{3} \mathrm{C}$ mejora la accesibilidad, disponibilidad e integración de estos recursos a otros. 
CBIE-LACLO 2015

Anais dos Workshops do IV Congresso Brasileiro de Informática na Educação (CBIE 2015)

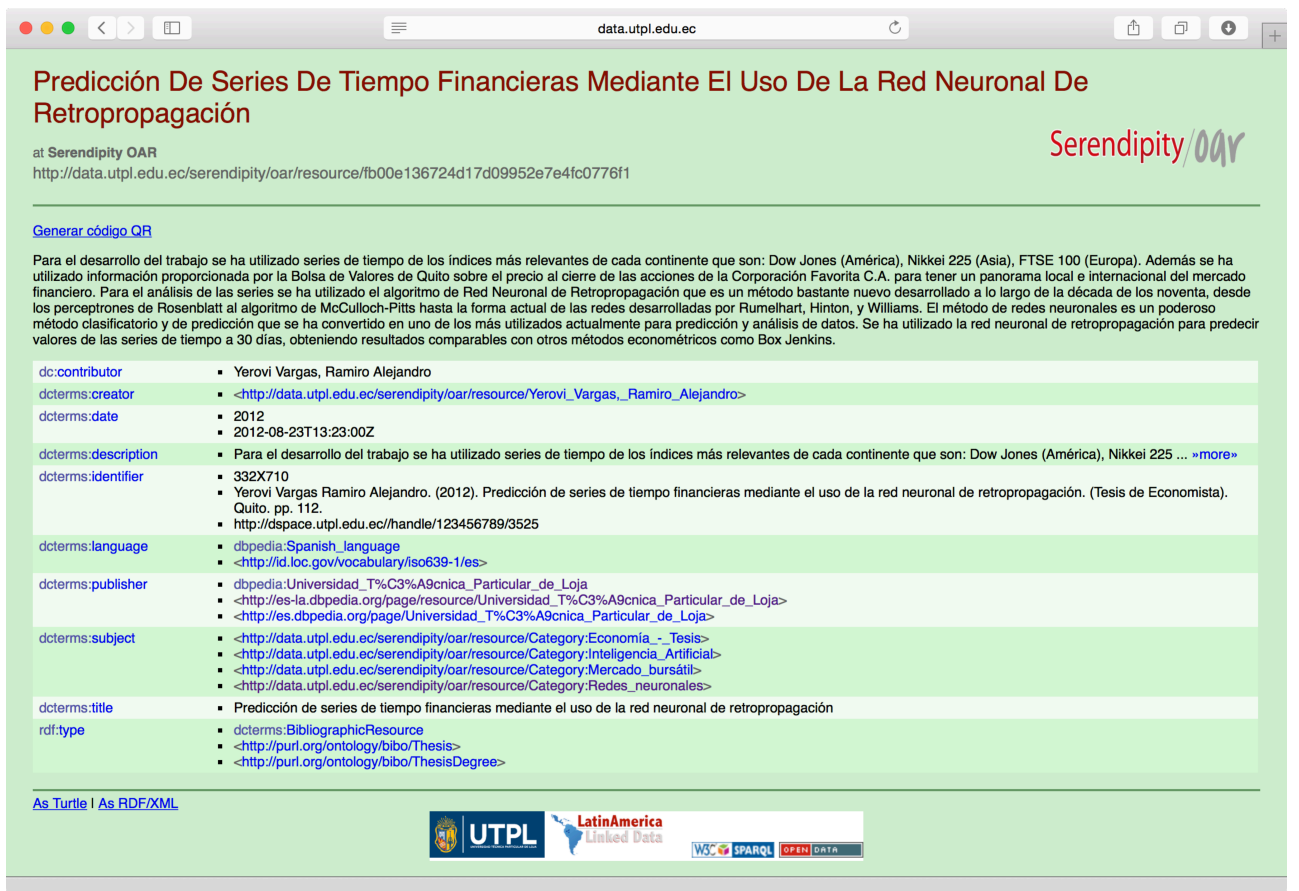

Figura 4 - Explorando datos enlazados en Serendipity OAR.

\section{Generación y Publicación de Datos Enlazados}

Finalmente, se realizó el proceso de generación de datos RDF es asignar URIs a los textos extraídos. Una vez que se han generado y almacenado los datos RDF, se ha habilitado un SParQL End Point para la consulta de datos, además se ha elegido a Pubby $^{3}$ como la interfaz Web para mostrar estos datos en formato legible para las personas (Ver Figura 4).

SPARQL endpoint: http://data.utpl.edu.ec/serendipity/oar/sparql

Graph: $\quad$ http://data.utpl.edu.ec/serendipity/oar

Ejemplo: http://data.utpl.edu.ec/serendipity/oar/resource/Category:Redes_neuronales

\section{Conclusiones y Trabajos Futuros}

En este trabajo, los autores se centran en el concepto de Web Semántica, desde una perspectiva del procesamiento de grandes volúmenes de datos enlazados (Linked Data) contenidos en repositorios digitales. En el contexto de Latinoamérica, el

${ }^{3}$ Pubby está disponible en: http://wifo5-03.informatik.uni-mannheim.de/pubby/ 
presente trabajo plantea un enfoque que mejora la integración de repositorios, y el soporte de estándares de metadatos formales y abiertos en la descripción de recursos educativos, a un nivel de especificidad, granularidad y complejidad realizable. De esta manera se busca mejorar el intercambio, el re-uso, la compartición, el enriquecimiento de datos y la colaboración institucional y académica en el contexto de librerías digitales. El modelo propuesto respeta y garantiza condiciones de autonomía local en un marco de interoperabilidad tecnológica global. El esfuerzo actual consiste en alcanzar acuerdos sobre la especificación de una contextualización de conceptos, así como en publicar e intercambiar datos enlazados en la Web. Se han examinado vocabularios para representar recursos digitales, clasificación de tópicos, descripción de organizaciones, autores, catálogos de datos y repositorios.

La adopción de un enfoque de integración e interoperabilidad semántica tiene el potencial de contribuir con: (a) la apertura en el movimiento de recursos educativos abiertos (Tovar, E. \& Piedra, N. 2014) y apoyar la identificación de recursos digitales y creación de rutas de aprendizaje; (b) el acceso a un conjunto más rico de información proveniente desde diferentes repositorios (búsquedas federadas); (c) a bibliotecarios y archivistas quienes tendrán acceso a datos compartidos por otros para describir sus recursos y reducir la redundancia; (e) a desarrolladores Web que enfrentan menores problemas de heterogeneidad en formatos y semántica de datos (Baker et al., 2011); (f) a investigadores que realizan extracción del conocimiento desde repositorios abiertos, por ejemplo para la búsqueda de expertos en un dominio determinado, detección de nuevos temas de investigación, análisis de redes de colaboración, (g) la integración de recursos digitales e información que está dispersa en la Red y que es clave para procesos de re-uso y adaptación, (f) la experiencia Web de búsqueda de información relacionada a la actividad de los docentes e investigadores universitarios; entre otros.

\section{Agradecimientos}

Este trabajo ha sido desarrollado con el apoyo de la Universidad Técnica Particular de Loja (UTPL) y el Consorcio Ecuatoriano para Desarrollo de Internet Avanzado (CEDIA) a través del Grupo de Trabajo de Repositorios Abiertos. Los autores agradecen el soporte recibido por el equipo de Tecnologías Avanzadas de la Web y Sistemas Basados en Conocimiento de la UTPL.

\section{Referencias Bibliográficas}

Anibaldi, S., Jaques, Y., Celli, F., Stellato, A., Keizer, J.(2015) Migrating bibliographic datasets to the Semantic Web: The AGRIS case. Semantic Web, 6(2), 113-120. 
Baker, T., Bermés, E., Coyle, k., Dunsire, G., Isaac, A., Murray, P., y otros (2011). Library Linked Data Incubator Group Final Report. Obtenido de http://www.w3.org/2005/Incubator/lld/XGR-lld20111025/\#Benefits_of_the_Linked_Data_Approach.

Berners-Lee, T. Hendler, J. Lassila, O. (2001). The Semantic Web, Scientific American, 284(5), 34-43.

Berners-Lee, Tim (2006). Linked Data-Design Issues. Obtenido de: http://www.w3.org/DesignIssues/LinkedData.html

Gendt, M.; Isaac, A.; Meij, L. \& Schlobach, S. (2006). Semantic Web Techniques for Multiple Views on Heterogeneous Collections: A Case Study. Research and Advanced Technology for Digital Libraries. Springer Berling Heidelberg, vol. 4172.

Francesconi, E.; Faro, S. ; Marinai, E. \& Perugi, G. (1008). A Methodological Framework for Thesaurus Semantic Interoperability. Proceeding of the Fifth European Semantic Web Conference, 76-87.

Heath, Tom \& Bizer, Christian (2011). Linked Data: Evolving the Web into a Global Data Space. Synthesis Lectures on the Semantic Web: Theory and Technology, 1(1), 1-136. Morgan \& Claypool.

IEEE (1990). IEEE Standard Glossary of Software Engineering Terminology. IEEE Standards Board, New York.

Lenzerini, M. (2002). Data Integration: A Theoretical Perspective. In Proceedings of the Twenty-first ACM SIGACT-SIGMOD-SIGART Symposium on Principles of Database Systems, June 3-5, Madison, Wisconsin, USA. doi: $10.1145 / 543613.543644$

Malakhov, D., Serebryakov, V., Teymurazov, K., Shorin, O. (2014) Semantic integration of bibliographic records. CEUR Workshop Proceedings, 35-41.

Piedra, N. Tovar, E. López, J. Chicaiza, J. (2014). Consuming and producing linked Open Data: The case of Opencourseware. Emerald EarlyCite. DOI: 10.1108/PROG-07-2012-0045

Piedra, N. Chicaiza, J. Quichimbo, P. Saquicela, V. Cadme, E. López, J. Espinoza, M. Tovar, E. (2015) Marco de Trabajo para la Integración de Recursos Digitales Basado en un Enfoque de Web Semántica. Revista Ibérica de Sistemas y Tecnologías de Información (RISTI). doi: 10.17013/risti.e3.55-70. http://www.scielo.mec.pt/pdf/rist/nspe3/nspe3ao6.pdf

OAI (2015). The Open Arvhives Initiative Protocolo for Metadata Harvesting, Implementation GuideLines. Obtenido de: http://www.openarchives.org/OAI/openarchivesprotocol.html.

Sztyler, T., Huber, J., Noessner, J., Murdock, J., Allen, C., Niepert, M.(2014). LODE: Linking digital humanities content to the web of data. Proceedings of the ACM/IEEE Joint Conference on Digital Libraries, 423-424.

Tovar, E. \& Piedra, N. (2014). Open Educational Resources in Engineering Education: a perspective to improve Reusability of Resources and Data. IEEE Transactions on Education, 57(4). DOI: http://10.1109/TE.2014.2359257

Zengenene, D., Casarosa, V., Meghini, C. (2014). Towards a Methodology for Publishing Library Linked Data. Communications in Computer and Information Science, 385 CCIS, 81-92. 